\title{
Minkowski Sum of a Voronoi Parallelotope and a Segment
}

\author{
Robert Erdahl $^{1}$, Viacheslav Grishukhin ${ }^{2, *}$ \\ ${ }^{1}$ Queen's University, Kingston, Canada \\ ${ }^{2}$ CEMI Russian Academy of Sciences,Nakhimovskii prosp.47 117418 Moscow, Russia
}

Copyright (C) 2015 by authors, all rights reserved. Authors agree that this article remains permanently

open access under the terms of the Creative Commons Attribution License 4.0 International License

\begin{abstract}
By a Voronoi parallelotope $P(a)$ we mean a parallelotope determined by linear in normal vectors $p$ inequalities with a non-negative quadratic form $a(p)$ as right hand side. For a positive form $a$, it was studied by Voronoi in his famous memoir. For a set of vectors $\mathcal{P}$, we call its dual a set of vectors $\mathcal{P}^{*}$ such that $\langle p, q\rangle \in\{0, \pm 1\}$ for all $p \in \mathcal{P}$ and $q \in \mathcal{P}^{*}$. We prove that Minkowski sum of an irreducible Voronoi parallelotope $P(a)$ and a segment $z(u)$ is a Voronoi parallelotope if and only if $u=w e$, where $w>0$ and $e$ is a vector of the dual of the set of normal vectors of all facets of $P(a)$. Then the segment $z(u)$ is described by the same set of inequalities with $w a_{e}(p)=w\langle e, p\rangle^{2}$ as right hand side and $P(a)+z(u)=P\left(a+w a_{e}\right)$. A similar assertion is true for Minkowski sum of a reducible Voronoi parallelotope with a segment.
\end{abstract}

Keywords Parallelotope, Voronoi Parallelotope, Minkowski Sum, Dual Set

\section{Introduction}

\subsection{Polytopes}

Consider a $d$-dimensional polytope $P(a)$ given by the following system of inequalities

$$
P(a, \mathcal{P})=\left\{x \in \mathbb{R}^{d}:\langle p, x\rangle \leq a(p) \text { for all } p \in \mathcal{P}\right\},
$$

where $\langle p, x\rangle$ is a scalar product of vectors $p, x \in \mathbb{R}^{d}$. Here $\mathcal{P} \subset \mathbb{R}^{d}$ is a set of vectors containing a set of normal vectors $\mathcal{P}_{n}$ of all facets of $P(a)$. If the set $\mathcal{P}$ spans the whole space $\mathbb{R}^{d}$, then $P(a, \mathcal{P})$ is a bounded polytope. The function $a: \mathcal{P} \rightarrow \mathbb{R}$ is an arbitrary function.

A polytope $P(a, \mathcal{P})$ is called reducible if it is a direct sum

$$
P(a, \mathcal{P})=P\left(a, \mathcal{P}_{1}\right) \oplus P\left(a, \mathcal{P}_{2}\right) \oplus \ldots \oplus P\left(a, \mathcal{P}_{k}\right)
$$

of polytopes $P\left(a, \mathcal{P}_{i}\right)$ such that the sets $\mathcal{P}_{i}$ span $d_{i^{-}}$ dimensional spaces $\mathbb{R}\left(\mathcal{P}_{i}\right), 1 \leq i \leq k$, that intersect in a point. We denote by $\mathcal{P}_{i n}$ a set of normal vectors of all facets of $P\left(a, \mathcal{P}_{i}\right)$. A polytope is called irreducible if it is not reducible.
For a vector $p \in \mathbb{R}^{d}$ and a number $a(p) \in \mathbb{R}$, we define the following affine hyperplane

$$
H_{p}(a)=\left\{x \in \mathbb{R}^{d}:\langle p, x\rangle=a(p)\right\}
$$

Call a face $F$ of the polytope $P(a, \mathcal{P})$ contact face and denote it $F(p)$ if $F=H_{p}(a) \cap P(a, \mathcal{P})$, i.e., if the hyperplane $H_{p}(a)$ supports $P(a, \mathcal{P})$ at the face $F$. Hence, we call the corresponding vector $p \in \mathcal{P}(a)$ contact vector and denote by $\mathcal{P}(a)$ the set of all contact vectors.

A special case of a polytope is a segment $z(e)$ of a line $l(e)$ spanned by a vector $e \in \mathbb{R}^{d}$, where

$$
z(e)=\left\{x \in \mathbb{R}^{d}: x=\lambda e,-1 \leq \lambda \leq 1\right\} .
$$

The segment $z(e)$ is symmetric with respect to origin 0 . We show below that $z(e)=P\left(f_{e}, \mathcal{P}\right)$ for some function $f_{e}(p)$ if the set $\mathcal{P}$ is good, i.e., it satisfies a special property (See Section "Segments").

\subsection{Parallelotopes}

We call the above $d$-dimensional polytope $P(a, \mathcal{P})$ a Voronoi parallelotope if the following conditions hold:

(i) the function $a(p)=\langle p, A p\rangle$ is a non-negative quadratic form;

(ii) the set $\mathcal{P}$ is symmetric, and the set $\mathcal{P}_{n}(a)$ of normal vectors generates integrally a $d$-dimensional lattice $L$ containing $\mathcal{P}$.

(iii) if $\operatorname{ker} A \neq \emptyset$, then $\operatorname{dim}(\operatorname{ker} A \cap \mathcal{P})=\operatorname{dim}(\operatorname{ker} A)$.

Here symmetric means that if $p \in \mathcal{P}$ then $-p \in \mathcal{P}$, too, and $\operatorname{ker} A$ denote kernel of the matrix $A$.

Recall that a parallelotope is a polytope whose parallel translations form a tiling, i.e. they fill its space without interstices (gaps) and intersections by inner points. Voronoi proved in Voronoi [1] that if the above conditions (i) with $a(p)$ positive and (ii) hold, then $P(a)$ is a parallelotope. Besides, if $a$ is positive, the parallelotope $P(a, \mathcal{P})$ is a Dirichlet-Voronoi cell of zero point 0 of the lattice $2 A L$ with respect to the metric form $a^{*}(q)=\frac{1}{2}\left\langle q, A^{-1} q\right\rangle$, i.e.

$P(a, \mathcal{P})=P\left(a^{*}\right)=\left\{x \in \mathbb{R}^{d}: a^{*}(x) \leq a^{*}(x-q)\right.$ for all $\left.q \in 2 A L\right\}$.

In fact, since $a^{*}(x-q)=a^{*}(x)-2 \frac{1}{2}\left\langle x, A^{-1} q\right\rangle+a^{*}(q)$ and $q=2 A p \in 2 A L$, the above inequalities are equivalent to 
$\left\langle x, A^{-1} 2 A p\right\rangle \leq \frac{1}{2}\left\langle 2 A p, A^{-1} 2 A p\right\rangle$, i.e. to $\langle p, x\rangle \leq a(p)$ for all $p \in L$.

One can prove that, for any Voronoi parallelotope $P(a, \mathcal{P})$, the set $\mathcal{P}$ can be enlarged up to a set $\mathcal{P}(a) \subset L$ of minimal (with respect to the form $a$ ) vectors of each parity class of $L$. Moreover, the whole lattice $L$ may be taken as the set $\mathcal{P}$. Note that only for $p \in \mathcal{P}(a)$ the hyperplane $H_{p}(a)$ supports the Voronoi parallelotope $P(a, \mathcal{P})$ at a face $F(p)$. For a parallelotope, each its contact face is an intersection of two parallelotopes. Dolbilin call in Dolbilin [2] faces with this property by standard faces.

For each $p \in \mathcal{P}(a)$, the vector $2 A p$ is called commensurate (with the parallelotope $P(a)$ ). The commensurate vector $2 A p$ connects the center of the parallelotope $P(a)$ with the center of a parallelotope that is adjacent to $P(a)$ by the contact face $F(p)$. Commensurate vectors generate the lattice $2 A L$.

In general, lattices $L$ and $2 A L$ are distinct. But, if $a(p)=$ $\frac{1}{2} p^{2}$, then the lattices $L$ and $2 A L$ coincide. In this case the Voronoi parallelotope is called usually Voronoi polytope, or Dirichlet-Voronoi cell.

Recall that the dual of a lattice $L$ is the lattice

$$
L^{*}=\left\{q \in \mathcal{R}^{d}:\langle q, p\rangle \in \mathbb{Z} \text { for all } p \in L\right\} .
$$

Since the set $\mathcal{P}_{n}(a)$ generates the lattice $L$, we can change $L$ by $\mathcal{P}_{n}(a)$ in the above definition of $L^{*}$. Define the following important subset $\mathcal{P}_{n}^{*}(a) \subset L^{*}$ as follows

$$
\mathcal{P}_{n}^{*}(a)=\left\{e \in \mathbb{R}^{d}:\langle e, p\rangle \in\{0, \pm 1\} \text { for all } p \in \mathcal{P}_{n}(a)\right\} .
$$

We call this set dual of $\mathcal{P}_{n}(a)$.

For a vector $e$, define the following quadratic form of rank 1

$$
a_{e}(p)=\langle p, e\rangle^{2},
$$

We prove below, that $z(e)=P\left(a_{e}, \mathcal{P}\right)$ is a parallelotope for a set $\mathcal{P}$ that is "very good" for $e$.

In this paper we prove the following

Theorem 1. Let $P(a, \mathcal{P})$ be a Voronoi parallelotope, defined in (1), where $\mathcal{P} \supseteq \mathcal{P}(a) \supseteq \mathcal{P}_{n}(a)$. Let $P(a, \mathcal{P})=\sum_{i=1}^{k} P\left(a, \mathcal{P}_{i}\right)$, where sum is direct and $P\left(a, \mathcal{P}_{i}\right)$ is an irreducible parallelotope for each $i$. Let $u \in \mathbb{R}^{d}$ be a vector. Then the following assertions are equivalent:

(i) Minkowski sum $P(a)+z(u)$ is a Voronoi parallelotope;

(ii) a projection of the vector $u$ on the space $\mathbb{R}\left(\mathcal{P}_{i}\right)$ is parallel to some vector $e_{i} \in \mathcal{P}_{i n}^{*}(a)$, i.e. $u=\sum_{i=1}^{k} w_{i} e_{i}$.

For $u=\sum_{i=1}^{k} w_{i} e_{i}$, we have

$P(a, \mathcal{P})+z(u)=P(a, \mathcal{P})+\sum_{i=1}^{k} w_{i} P\left(a_{e_{i}}, \mathcal{P}\right)=P\left(a+\sum_{i=1}^{k} w_{i} a_{e_{i}}, \mathcal{P}\right)$

The implication $(i i) \Rightarrow(i)$ was proved in Grishukhin [3].

Magazinov proved in Magazinov [4] that (in our terms) $P(a)+b z(e)$ is a Voronoi parallelotope if this sum is a parallelotope. It seems to us that our proof is simpler.

Note that the quadratic form $a_{e}(p)=\langle p, e\rangle^{2}$ of rank 1 is not positive. If we consider $e$ as a column vector, then $A_{e}=e e^{T}$ is Gram matrix of the form $a_{e}$. It transforms vectors $p$ of the lattice $L$ generated by $\mathcal{P}$ into $A_{e} p=e\langle e, p\rangle$ that is projection of $p$ onto the line $l(e)$ spanned by $e$. The kernel $\operatorname{ker} A_{e}$ is the hyperplane $H_{e}(0)$. By condition (iii), $P\left(a_{e}, \mathcal{P}\right)$ is a parallelotope if the intersection $\mathcal{P} \cap H_{e}(0)$ generates a $(d-1)$-dimensional lattice. This lattice determines a partition of $L$ into layers.

Hence $A_{e} L$ is projection of $L$ onto $l(e)$. If $L=\sum_{i=1}^{k} L_{i}$, then projection $A_{e} L_{i}$ is a lattice if and only if, for all $p \in L_{i}$, $\langle e, p\rangle=w_{i} k(p)$, where $k(p) \in \mathbb{Z}$ is an integer, and $w_{i} \in \mathbb{R}$ does not depend on $p$. We see that this condition holds if condition (ii) of Theorem 5 holds.

\section{Minkowski sum of polytopes}

For a fixed set $\mathcal{P}$ of vectors, the polytopes $P(a, \mathcal{P})$ defined in (1) have the following simple property

Lemma 1. For any functions $a_{1}(p)$ and $a_{2}(p)$, the following inclusion holds.

$$
P\left(a_{1}, \mathcal{P}\right)+P\left(a_{2}, \mathcal{P}\right) \subseteq P\left(a_{1}+a_{2}, \mathcal{P}\right) .
$$

If $p \in \mathcal{P}$ is a contact vector of both the polytopes $P\left(a_{1}, \mathcal{P}\right)$ and $P\left(a_{2}, \mathcal{P}\right)$, then $p$ is a contact vector of the sum $P\left(a_{1}, \mathcal{P}\right)+$ $P\left(a_{2}, \mathcal{P}\right)$ and of the polytope $P\left(a_{1}+a_{2}, \mathcal{P}\right)$.

Proof. For $k \in\{1,2\}$, let $x_{k} \in P\left(a_{k}, \mathcal{P}\right)$. Then $\left\langle p, x_{k}\right\rangle \leq$ $a_{k}(p)$ for all $p \in \mathcal{P}$. This implies that $\left\langle p,\left(x_{1}+x_{2}\right)\right\rangle \leq a_{1}(p)+$ $a_{2}(p)$ for all $p \in \mathcal{P}$, i.e., $x_{1}+x_{2} \in P\left(a_{1}+a_{2}\right)$. Hence $P\left(a_{1}\right)+$ $P\left(a_{2}\right) \subseteq P\left(a_{1}+a_{2}\right)$.

If $p$ is a contact vector of both the polytopes $P\left(a_{k}, \mathcal{P}\right), k=$ 1,2 , then the above two inequalities with $x_{k}$ hold as equalities. Hence $\left\langle p, x_{1}+x_{2}\right\rangle=a_{1}(p)+a_{2}(p), H_{p}\left(a_{1}+a_{2}\right)$ supports the sum of polytopes, and, therefore, $p$ is a contact vector of the sum. Obviously, the intersection $P\left(a_{1}+a_{2}, \mathcal{P}\right) \cap H_{p}\left(a_{1}+a_{2}\right)=F(p)$ is a contact face of $P\left(a_{1}+a_{2}, \mathcal{P}\right)$.

It is a problem to find conditions when the equality $P\left(a_{1}, \mathcal{P}\right)+P\left(a_{2}, \mathcal{P}\right)=P\left(a_{1}+a_{2}, \mathcal{P}\right)$ holds. This equality does not hold if the sum $P\left(a_{1}, \mathcal{P}\right)+P\left(a_{2}, \mathcal{P}\right)$ contains a facet that is not a facet of $P\left(a_{1}+a_{2}, \mathcal{P}\right)$. Hence Lemma 2 below gives a sufficient condition for the equality $P\left(a_{1}, \mathcal{P}\right)+P\left(a_{2}, \mathcal{P}\right)=$ $P\left(a_{1}+a_{2}, \mathcal{P}\right)$.

Lemma 2. Suppose that each facet of the sum $P\left(a_{1}, \mathcal{P}\right)+$ $P\left(a_{2}, \mathcal{P}\right)$ is determened by a contact vector $p \in \mathcal{P}$. Then

$$
P\left(a_{1}, \mathcal{P}\right)+P\left(a_{2}, \mathcal{P}\right)=P\left(a_{1}+a_{2}, \mathcal{P}\right)
$$

Proof. The condition of this Lemma means that each facet $F$ of the sum $P\left(a_{1}, \mathcal{P}\right)+P\left(a_{2}, \mathcal{P}\right)$ has the form $F=F_{1}(p)+$ $F_{2}(p)$, where $F_{k}(p)$ is a contact face of $P\left(a_{k}, \mathcal{P}\right)$ for both $k=$ 1,2 . Let $\mathcal{P}_{n} \subseteq \mathcal{P}$ be a set of normal vectors of all facets of the sum $P\left(a_{1}, \mathcal{P}\right)+P\left(a_{2}, \mathcal{P}\right)$. Then the following system of inequalities

$$
\langle p, x\rangle \leq a_{1}(p)+a_{2}(p) \text { for all } p \in \mathcal{P}_{n}
$$

desribes the sum $P\left(a_{1}, \mathcal{P}\right)+P\left(a_{2}, \mathcal{P}\right)$. But this is equivalent to the equality

$$
P\left(a_{1}, \mathcal{P}\right)+P\left(a_{2}, \mathcal{P}\right)=P\left(a_{1}+a_{2}, \mathcal{P}_{n}\right) .
$$

By Lemma 1, inequalities $\langle p, x\rangle \leq a_{1}(p)+a_{2}(p)$ are feasible for the sum $P\left(a_{1}, \mathcal{P}\right)+P\left(a_{2}, \mathcal{P}\right)$ for all $p \in \mathcal{P}$. This implies that $P\left(a_{1}+a_{2}, \mathcal{P}_{n}\right)=P\left(a_{1}+a_{2}, \mathcal{P}\right)$.

We show below that the equality $P\left(a_{1}, \mathcal{P}\right)+P\left(a_{2}, \mathcal{P}\right)=$ $P\left(a_{1}+a_{2}, \mathcal{P}\right)$ holds when $P\left(a_{2}\right)$ is a segment $z(e)$ and $a_{2}=f_{e}$, where the function $f_{e}(p)$ is defined below in (6).

For $i=1,2$, let $a_{i}$ be a non-negative quadratic form and $P\left(a_{i}, \mathcal{P}\right)$ be the corresponding Voronoi parallelotope described in (1). It is also a problem to find conditions when the sum $P\left(a_{1}, \mathcal{P}\right)+P\left(a_{2}, \mathcal{P}\right)$ is a parallelotope, and, in particular, it is a Voronoi parallelotope. 
It is shown in Ryshkov at al [5] that the equality $P\left(a_{1}, \mathcal{P}\right)+$ $P\left(a_{2}, \mathcal{P}\right)=P\left(a_{1}+a_{2}, \mathcal{P}\right)$ holds if $a_{1}$ and $a_{2}$ belong to closure of an L-type domain. We show below that this equality holds if $a_{2}(p)=a_{e}(p)$, where the quadratic form $a_{e}=\langle p, e\rangle^{2}$ of rank 1 relates to the segment $z(e)$, and then the sum $P\left(a_{1}, \mathcal{P}\right)+$ $P\left(a_{e}, \mathcal{P}\right)$ is a parallelotope.

\section{Segments}

Let $e, p \in \mathbb{R}^{d}$. Consider the affine hyperplane $H_{p}\left(f_{e}\right)$ defined in (2), where

$$
f_{e}(p)=\frac{\langle p, e\rangle^{2}}{|\langle p, e\rangle|}
$$

It is natural to suppose that $f_{e}(p)=0$ if $\langle p, e\rangle=0$.

Lemma 3. For any vector $p \in \mathbb{R}^{d}$, the hyperplane $H_{p}\left(f_{e}\right)$ supports the segment $z(e)$.

Proof. Note that end-vertices of the segment $z(e)$ are points $\pm e$. If $\langle p, e\rangle>0$, then the end-vertex $e$ lies on $H_{p}\left(f_{e}\right)$. If $\langle p, e\rangle<0$, then the end-vertex $-e$ lies on $H_{p}\left(f_{e}\right)$. If $\langle p, e\rangle=0$, then the whole segment $z(e)$ lies on $H_{p}\left(f_{e}\right)$.

We say that a set of vectors $\mathcal{P}$ is $\operatorname{good}$ for a vector $e$, if the following conditions hold:

(i) scalar products $\langle p, e\rangle$ have all the three signs,+- and 0 , for all $p \in \mathcal{P}$;

(ii) the polytope $P\left(0, \mathcal{P}_{0}(e)\right)$, where $\mathcal{P}_{0}(e)=\{p \in \mathcal{P}:\langle p, e\rangle=$ $0\}$, is a line (spanned by $e$ ).

Lemma 3 implies the following fact.

Lemma 4. Let the function $f_{e}(p)$ is defined in (6), $P\left(f_{e}, \mathcal{P}\right)$ is $P(a, \mathcal{P})$ for $a=f_{e}, P(a, \mathcal{P})$ is given by $(1)$ and $\mathcal{P}$ is good for $e$. Then

$$
z(e)=P\left(f_{e}, \mathcal{P}\right) .
$$

Proof. Note that $f_{e}(p)=0$ for $p \in \mathcal{P}_{0}(e)$. Since $P\left(0, \mathcal{P}_{0}(e)\right)$ is a line spanned by the vector $e$, the conditions of this Lemma and Lemma 3 imply that $z(e)=P\left(f_{e}, \mathcal{P}\right)$.

\section{Minkowski sum of a polytope with a segment}

At first, we consider the Minkowski sum $P(a, \mathcal{P})+z(e)$ of an arbitrary polytope $P=P(a, \mathcal{P})$ defined in (1) with the segment $z(e)$ defined in (3). Á.Horváth call in Horváth [6] the sum $P+z(e)$ by an extension $P^{e}$ of $P$. Recall that we call a face $F$ contact and denote it by $F(p)$ if $F=P(a, \mathcal{P}) \cap H_{p}(a)$. The vector $p$ is called contact vector of the face $F=F(p)$.

For a face $F$ of a polytope $P=P(a, \mathcal{P})$, let $l_{F}(e)$ be a parallel shift of the line $l(e)$ (spanned by $e$ ) such that $l_{F}(e) \cap F \neq \emptyset$. Call the face $F$ transversal to $e$ if $l_{F}(e) \cap F$ is a point. Otherwise, call the face $F$ parallel to $e$ and denote this fact as $F \| e$. If $F=F(p)$ is a contact face of $P(a, \mathcal{P})$, then $F(p) \| e$ implies $l_{F}(e) \subset H_{p}(a)$.

We say that a face $F$ belongs to a shadow boundary of $P$ in direction $e$ if $l_{F}(e) \cap F=l_{F}(e) \cap P$. Denote by $\mathcal{F}_{e}(P)$ a set of all faces of $P$ that belong to the shadow boundary of $P$ in direction of $e$. It is worth to note that faces of the shadow boundary are considered as open faces. Let $F \in \mathcal{F}_{e}(P)$ and $F^{\prime} \subset F$ be a subface of $F$. If $l_{F^{\prime}}(e)=l_{F}(e)$ and $l_{F^{\prime}}(e) \cap$
$F^{\prime} \neq l_{F^{\prime}}(e) \cap P$, then $F^{\prime} \notin \mathcal{F}_{e}(P)$. If there is $l_{F^{\prime}} \neq l_{F}$ and $l_{F^{\prime}}(e) \cap F^{\prime}=l_{F^{\prime}}(e) \cap P$, then $F^{\prime} \in \mathcal{F}_{e}(P)$.

A face $F$ is transformed into a face $F+z(e)$ in the extension $P^{e}=P+z(e)$. Denote dimension of $F$ by $\operatorname{dim} F$. Lemma 5 below helps to understand how faces of $P^{e}$ change with respect to faces of $P$. Assertions of Lemma 5 are obvious.

Lemma 5. Let $F$ be a face of a polytope P. Consider the sum $P^{e}=P+z(e)$. There are the following three possibilities for the sum $F+z(e)$ :

(i) if $F$ is parallel to $e$, then $F+z(e)=F^{e}$ is an extension of $F$, and $\operatorname{dim}(F+z(e))=\operatorname{dim} F$;

(ii) if $F$ is transversal to $e$ and $F \notin \mathcal{F}_{e}(P)$, then $F+z(e)=$ $F+e$ is a parallel shift of $F$ by the vector $e$;

(iii) if $F$ is transversal to $e$ and $F \in \mathcal{F}_{e}(P)$, then $F+z(e)=$ $F \oplus z(e)$ is direct sum of $F$ and $z(e)$, and $\operatorname{dim}(F \oplus z(e))=$ $\operatorname{dim} F+1$.

Now consider Minkowski sum of a polytope $P(a, \mathcal{P})$ given in (1) and a segment $z(e)$. Suppose that the set $\mathcal{P}$ is good for $e$. Then, by Lemma $4, z(e)=P\left(f_{e}, \mathcal{P}\right)$. If we want to prove that $\left.P(a, \mathcal{P})+P\left(f_{e}, \mathcal{P}\right)=P\left(a+f_{e}\right), \mathcal{P}\right)$, then, according to Lemma 2, we have to prove that each facet of the sum $P(a, \mathcal{P})+P\left(f_{e}, \mathcal{P}\right)$ is determined by a contact vector $p \in \mathcal{P}$.

Lemma 6. Let $P=P(a, \mathcal{P})$ be a polytope, where the set $\mathcal{P}$ is good for a vector . Suppose that each $(d-2)$-face $F \in \mathcal{F}_{e}(P)$, which is transversal to $e$, is a contact face $F=F(p)$ such that $\langle e, p\rangle=0$. Then each facet of the sum $P(a, \mathcal{P})+P\left(f_{e}, \mathcal{P}\right)$ is supported by the hyperplane $H_{p}\left(a+f_{e}\right)$ for some $p \in \mathcal{P}$.

Proof. By Lemma 4, we have $z(e)=P\left(f_{e}, \mathcal{P}\right)$. According to Lemma 5, each facet $F_{e}$ of the sum $P^{e}(a)=P(a)+z(e)$ has one of the following three types

(i) extension $F_{e}(p)=F^{e}(p)$ of a facet $F(p)$ of $P$;

(ii) a parallel shift $F_{e}(p)=F(p)+e$ of a facet $F(p)$ of $P$;

(iii) direct sum $F_{e}(p)=F(p) \oplus z(e)$ of a $(d-2)$-face $F(p)$ of $P$ and the segment $z(e)$.

Consider the three cases (i), (ii) and (iii).

Case (i). In this case, $F(p) \| e$. Since $F(p)$ lies on the hyperplane $H_{p}(a)$, we have $e \| H_{p}(a)$. This implies that $\langle p, e\rangle=0$, and therefore $f_{e}(p)=0$. Hence the facet $F_{e}(p)=(F(p))^{e}$ lies in the hyperplane $H_{p}(a)=H_{p}\left(a+f_{e}\right)$.

Case (ii). Let the facet $F(p)$ of $P$ be transversal to $e$ and $F(p) \notin \mathcal{F}_{e}(P)$. Then $\langle p, e\rangle \neq 0$. Hence the sum $F(p)+e$ is a shift of $F(p)$ obtained as follows. Let $x \in F(p)$. Then the point

$$
x+e \frac{\langle p, e\rangle}{|\langle p, e\rangle|}
$$

belongs to $F(p)+e$. Here the multiple $\frac{\langle p, e\rangle}{\langle p, e\rangle}$ describes direction of the shift. Since $F(p)$ is a facet of $P=P(a, \mathcal{P})$, we have $\langle p, x\rangle=a(p)$ and therefore

$$
\left\langle p, x+e \frac{\langle p, e\rangle}{|\langle p, e\rangle|}\right\rangle=a(p)+f_{e}(p)=\left(a+f_{e}\right)(p) .
$$

Since $x$ is an arbitrary point of $F(p)$, this implies that the facet $F_{e}(p)=F(p)+e$ of $P+z(e)$ is supported by $H_{p}\left(a+f_{e}\right)$.

Case (iii). Let $F(p)$ be a contact $(d-2)$-face of $P$ that is transversal to $e$ and $F(p) \in \mathcal{F}_{e}(P)$. The face $F(p)$ is transformed into the facet $F_{e}(p)=F(p) \oplus z(e)$ of $P+z(e)$. Since $\langle p, e\rangle=0$, the hyperplane $H_{p}(a)=H_{p}\left(a+f_{e}\right)$ supports the face $F_{e}(p)=F(p) \oplus z(e)$.

Now Lemma 6 and Lemma 2 imply the following 
Theorem 2. Let $P=P\left(a, \mathcal{P}_{a}\right)$ be a polytope and $z(e)=$ $P\left(f_{e}, \mathcal{P}\right)$ be a segment, where the set $\mathcal{P}$ is good for $e$ and $\mathcal{P}_{a} \subseteq \mathcal{P}$. Let each $(d-2)$-face $F \in \mathcal{F}_{e}(P)$ that is transversal to e be a contact face $F(p)$ of $P=P\left(a, \mathcal{P}_{a}\right)$ for $p \in \mathcal{P}_{a}$ such that $\langle p, e\rangle=0$. Then

$$
P+z(e)=P\left(a, \mathcal{P}_{a}\right)+P\left(f_{e}, \mathcal{P}\right)=P\left(a+f_{e}, \mathcal{P}_{a}\right) .
$$

Proof. If $\mathcal{P}_{a}$ is good for $e$, without loss of generality, we can set $\mathcal{P}=\mathcal{P}_{a}$. If $\mathcal{P}_{a}$ is not good for $e$, we can enlarge the set $\mathcal{P}_{a}$ up to the set $\mathcal{P}$ such that right-hand sides $a(p)$ for new vectors $p \in \mathcal{P}-\mathcal{P}_{a}$ are chosen such that the halfspaces $\left\{x \in \mathbb{R}^{d}:\langle p, x\rangle \leq a(p)\right\}$ contain $P\left(a, \mathcal{P}_{a}\right),\langle p, e\rangle=0$ and the hyperplane $H_{p}(a)$ supports $P$. Hence $P\left(a, \mathcal{P}_{a}\right)=P(a, \mathcal{P})$.

Now Lemmas 6 and 2 imply the following equality

$$
P+z(e)=P(a, \mathcal{P})+P\left(f_{e}, \mathcal{P}\right)=P\left(a+f_{e}, \mathcal{P}\right) .
$$

Note that the hyperplanes $H_{p}(a)=H_{p}\left(a+f_{e}\right)$ for $p \in \mathcal{P}-\mathcal{P}_{a}$ support neither facets nor $(d-2)$-faces of $P=P\left(a, \mathcal{P}_{a}\right)$ that are transversal to $e$. Hence $P\left(a+f_{e}, \mathcal{P}\right)=P\left(a+f_{e}, \mathcal{P}_{a}\right)$. So, we have the wanted equality.

Remark. It is worth to note that Theorem 2 demands only that $\mathcal{P}_{a}$ is contained in the set $\mathcal{P}$ that is good for $e$. But the set $\mathcal{P}_{a}$ can be contained in $\mathcal{P}$ strictly.

\section{Minkowski sum of a Voronoi paral- lelotope with a segment}

Now we consider Minkowski sum of a Voronoi parallelotope $P(a, \mathcal{P})$ and the segment $z(u)$ for some vector $u$. We suppose that $\mathcal{P}$ contains the set of contact vectors of all contact faces of $P(a)$. At first, we suppose that $P(a, \mathcal{P})$ is irreducible.

Obviously, each segment is a parallelotope, and moreover it is a Voronoi parallelotope $P\left(a_{u}, \mathcal{P}\right)$. In fact, we can choose lengths of vectors $p \in \mathcal{P}$ such that $\langle p, u\rangle \in\{0, \pm 1\}$. If $\mathcal{P}$ is good for $u$, the function $f_{u}(p)$ is transformed in the quadratic form $a_{u}(p)$ defined in $(5)$, and $P\left(a_{u}, \mathcal{P}\right)$ is a Voronoi parallelotope.

But when we consider a sum of a parallelotope $P(a, \mathcal{P})$ with a segment $z(u)$, we cannot change lengths of vectors $p \in \mathcal{P}$. We can represent $z(u)$ in the form $P\left(f_{u}, \mathcal{P}\right)$ only if the set $\mathcal{P}$ is good for $u$. In the case of a parallelotope $P(a, \mathcal{P})$, we change the notion "good" by notion "very good" as follows.

We say that a symmetric set of vectors $\mathcal{P}(e)$ is very good for a vector $e$, if the following conditions hold:

(i) scalar products $\langle p, e\rangle$ takes all the three values \pm 1 and 0 , for all $p \in \mathcal{P}(e)$;

(ii) the set $\mathcal{P}_{0}(e)=\{p \in \mathcal{P}(e):\langle p, e\rangle=0\}$ spans a hyperplane $H_{e}(0)$.

The conditions (ii) of notions "good" and "very good" are equivalent, since in the last case the set $\mathcal{P}(e)$ is symmetric. Hence the notion "very good" is a strengthening of the notion "good".

It is worth to note that (ii) above is equivalent to item (iii) of definition (see Introduction) of the Voronoi parallelotope $P\left(a_{e}, \mathcal{P}\right)$.

Let $\mathcal{P}_{n}(a) \subseteq \mathcal{P}(a)$ be a set of normal vectors of facets of $P(a, \mathcal{P})$. If, for the vector $u \in \mathbb{R}^{d}$, the inclusions $\langle p, u\rangle \in$ $\{0, \pm w\}$ hold for all $p \in \mathcal{P}_{n}(a)$, then one can set $u=w e$ for $e$ such that $\langle p, e\rangle \in\{0, \pm 1\}$ for all $p \in \mathcal{P}_{n}(a)$. Hence we will consider vectors $e \in \mathcal{P}_{n}^{*}(a)$, where the dual $\mathcal{P}_{n}^{*}(a)$ is defined in (4). Recall that the set $\mathcal{P}_{n}(a)$ generates a $d$-dimensional lattice $L$.

Of course, there may be another vectors $p \in \mathcal{P}$ with $\langle p, e\rangle \in$ $\{0, \pm 1\}$. Hence we introduce the following set

$$
\mathcal{P}_{e}(a)=\{p \in \mathcal{P}(a):\langle p, e\rangle \in\{0, \pm 1\}\} .
$$

Obviously, $\mathcal{P}_{n}(a) \subseteq \mathcal{P}_{e}(a) \subseteq \mathcal{P}(e)$, where, recall, $\mathcal{P}(e)$ is a very good for $e$ set.

Let a parallelotope $P(a, \mathcal{P})$ be given by (1). Then, for $w>$ 0 , we have

$$
w P(a, \mathcal{P})=P(w a, \mathcal{P})
$$

Lemma 7. Let $u=w e$, where $w>0$ and $e \in \mathcal{P}_{n}^{*}(a)$. Let $p \in \mathcal{P}(e)$. Then $z(u)=w z(e)$,

$f_{e}(p)=\langle p, e\rangle^{2}=a_{e}(p)$, and $z(u)=w P\left(a_{e}, \mathcal{P}(e)\right)=P\left(w a_{e}, \mathcal{P}(e)\right)$

where the function $f_{e}(p)$ is defined in (6).

Proof. It is easy to see that $f_{e}(p)=a_{e}(p)$ for all $p \in \mathcal{P}(e)$. By Lemma 4, $z(e)=P\left(a_{e}, \mathcal{P}(e)\right)$. Using (8), we obtain last equalities.

Lemma 8. Let $\mathcal{P}_{n}($ a) be a set of normal vectors of a Voronoi parallelotope $P=P(a, \mathcal{P})$. Let $e \in \mathcal{P}_{n}^{*}(a)$, and let $F \in \mathcal{F}_{e}(P)$ be $a(d-2)$-face of $P$ that is transversal to $e$. Then $F=F(p)$ is a contact face for a contact vector $p$ such that $\langle p, e\rangle=0$.

Proof. Suppose to the contrary that $F$ generates a 6-belt $B$. Let $\pm p_{1}, \pm p_{2}, \pm p_{3} \in \mathcal{P}_{n}(a)$ be normal vectors of the 6-belt $B$. Let $F=F\left(p_{1}\right) \cap F\left(p_{2}\right)$. Note that $F\left(p_{1}\right), F\left(p_{2}\right) \notin \mathcal{F}_{e}(P)$, since $F$ is transversal to $e$ and $F \in \mathcal{F}_{e}(P)$. Hence, for $i=1,2$, $\left\langle p_{i}, e\right\rangle \neq 0$, and therefore $\left\langle p_{i}, e\right\rangle \in\{ \pm 1\}$. Without loss of generality, we can suppose that $\left\langle p_{1}, e\right\rangle=1$ and $\left\langle p_{2}, e\right\rangle=-1$. Let $F\left(p_{3}\right) \neq F\left(p_{2}\right)$ be the other facet of the 6-belt $B$ that is adjacent to $F\left(p_{1}\right)$. Since $P=P(a, \mathcal{P})$ is a Voronoi parallelotope, the equality $p_{3}=p_{1}-p_{2}$ holds. This equality implies the equality $\left\langle p_{3}, e\right\rangle=\left\langle p_{1}, e\right\rangle-\left\langle p_{2}, e\right\rangle=2$ that contradicts to $\left\langle p_{3}, e\right\rangle \in\{0, \pm 1\}$. Hence $F$ cannot generate a 6 -belt. Therefore $F=F(p)$ is a contact face.

Obviously, $F(p)=F\left(p_{1}\right) \cap F\left(p_{2}\right)$, where $F\left(p_{1}\right), F\left(p_{2}\right)$ are facets of $P(a, \mathcal{P})$. Recall that $2 A p_{1}$ and $2 A p_{2}$ are commensurate vectors of facets $F\left(p_{1}\right)$ and $F\left(p_{2}\right)$. Hence the vector $2 A\left(p_{1}+p_{2}\right)$ is a commensurate vector of the contact face $F(p)$. Therefore $p=p_{1}+p_{2}$. Since $e \in \mathcal{P}_{n}^{*}(a),\left\langle p_{1}, e\right\rangle,\left\langle p_{2}, e\right\rangle \in\{0, \pm 1\}$. If $\left\langle p_{1}, e\right\rangle=\left\langle p_{2}, e\right\rangle=0$, then $\langle p, e\rangle=0$. Otherwise, without loss of generality, we can suppose that $\left\langle p_{1}, e\right\rangle=1,\left\langle p_{2}, e\right\rangle=-1$. this implies $\langle p, e\rangle=0$.

Note that Lemma 8 implies that $p \in \mathcal{P}_{e}(a)$ for all contact vectors of $(d-2)$-faces $F(p) \in \mathcal{F}_{e}(P)$. Recall that $\mathcal{P}_{n}(a) \subseteq$ $\mathcal{P}_{e}(a)$.

Theorem 3. For $\mathcal{P} \supseteq \mathcal{P}(a)$, let $P=P(a, \mathcal{P})$ be a Voronoi parallelotope defined in (1), where $\mathcal{P}_{n}(a) \subseteq \mathcal{P}_{e}(a) \subseteq \mathcal{P}(e)$, and $\mathcal{P}(e)$ is a very good for e set. Then

$$
P(a, \mathcal{P})+z(e)=P\left(a, \mathcal{P}_{e}(a)\right)+P\left(a_{e}, \mathcal{P}(e)\right)=P\left(a+a_{e}, \mathcal{P}\right) .
$$

Proof. Let $F(p) \in \mathcal{F}_{e}(P)$ be a $(d-2)$-face that is transversal to $e$. Then, by Lemma $8, F=F(p)$ is a contact face of $P=$ $P(a, \mathcal{P})$ and $\langle p, e\rangle=0$. Since $a_{e}(p)=f_{e}(p)$ for all $p \in \mathcal{P}(e)$, we can apply Theorem 2 , where $\mathcal{P}_{a}=\mathcal{P}_{e}(a)$ and $\mathcal{P}=\mathcal{P}(e)$. We obtain $P\left(a, \mathcal{P}_{e}(a)\right)+z(e)=P\left(a+a_{e}, \mathcal{P}_{e}(a)\right)$. Note that new normal vectors of the sum $P+z(e)$ are obtained from above contact vectors of $(d-2)$-faces $F \in \mathcal{F}_{e}(P)$. Hence the set 
$\mathcal{P}_{e}(a) \subseteq \mathcal{P}$ contains all normal vectors of the sum $P+z(e)$, and $P\left(a+a_{e}, \mathcal{P}_{e}(a)\right)=P\left(a+a_{e}, \mathcal{P}\right)$.The assertion of this Theorem holds.

So, we have proved that $P(a, \mathcal{P})+P\left(a_{e}, \mathcal{P}\right)=P\left(a+a_{e}, \mathcal{P}\right)$ if $e \in \mathcal{P}_{n}^{*}(a)$ and $\mathcal{P} \supseteq \mathcal{P}_{e}(a)$. Recall that $a_{e}(p)=\langle e, p\rangle^{2}$. Now we will prove the following

Theorem 4. If the Voronoi parallelotope $P(a, \mathcal{P})$ is irreducible and the sum $P(a, \mathcal{P})+z(u)$ is a parallelotope, then $u=$ we for some $e \in \mathcal{P}_{n}^{*}(a)$ and $P(a, \mathcal{P})+z(u)=P\left(a+w a_{e}, \mathcal{P}\right)$.

A proof of Theorem 4 is based on two Lemmas below. Let

$$
\mathcal{P}_{0}(u)=\left\{p \in \mathcal{P}_{n}(a):\langle p, u\rangle=0\right\}
$$

and

$$
\mathcal{P}_{1}(u)=\left\{p \in \mathcal{P}_{n}(a):\langle p, u\rangle>0\right\} .
$$

Note that the sets $2 A \mathcal{P}_{0}(u)$ and $\pm 2 A \mathcal{P}_{1}(u)$ are sets of facet vectors of facets $F$ of $P=P(a, \mathcal{P})$ that belong and do not belong to shadow boundary $\mathcal{F}_{u}(P)$, respectively. Let $L_{0}(u)$ be a lattice integrally generated by vectors of the set $\mathcal{P}_{0}(u)$.

Obviously, the parallelotope $P^{u}=P(a, \mathcal{P})+z(u)$ has a nonzero width in direction of the line $l(u)$. Let $\mathcal{C}\left(P^{u}\right)$ be the set of commensurate (facet) vectors of facets of $P^{u}$ that belong to shadow boundary of $P(a, \mathcal{P})$ in direction of $l(u)$. Facet vectors of $\mathcal{C}\left(P^{u}\right)$ are either facet vectors of $P(a, \mathcal{P})$ of facets that belong to shadow boundary, or commensurate vectors of contact $(d-2)$-faces of that shadow boundary. B.Venkov proved in Venkov [7] that vectors of $\mathcal{C}\left(P^{u}\right)$ generate a $(d-1)$ dimensional lattice $L_{u}$. Obviously, $2 A L_{0}(u) \subseteq L_{u}$. Hence $\operatorname{dim} L_{0}(u) \leq d-1$. Lemma 9 below shows that the equality $\operatorname{dim} L_{0}(u)=d-1$ holds if $P(a, \mathcal{P})$ is irreducible.

Lemma 9. If the Voronoi parallelotope $P(a, \mathcal{P})$ is irreducible, then dimension of the lattice $L_{0}(u)$ is $d-1$.

Proof. It is proved in Grishukhin [3] and Dutour at al [9] that the set $\mathcal{P}_{0}(u)$ intersects triples of normal vectors of all 6-belt of $P(a, \mathcal{P})$ if the sum $P(a, \mathcal{P})+z(u)$ is a parallelotope. Consider the space $H_{0}=\cap_{p \in \mathcal{P}_{0}(u)} H_{p}(0)$. A.Magazinov called the space $H_{0}$ perfect. For each vector $v \in H_{0}$, the sum $P(a, \mathcal{P})+z(v)$ is a parallelotope. It is obvious that $u \in H_{0}$. A.Magazinov proved in Magazinov [4], Theorem 4.4, that the parallelotope $P(a, \mathcal{P})$ is reducible if dimension of the space $H_{0}$ is greater than 1 . This implies that, for the irreducible Voronoi parallelotope $P(a, \mathcal{P})$, the space $H_{0}$ is the line $l(u)$, and the set $\mathcal{P}_{0}(u)$ generates a hyperplane $H(u)$ that is orthogonal to the vector $u$. The lattice $L_{0}(u) \subseteq L \cap H(u)$ has dimension $d-1$, since $\mathcal{P}_{0}(u)$ generates it.

Let $L_{1}(u)$ be a lattice integrally generated by differences $p-p^{\prime}$ of vectors $p, p^{\prime} \in \mathcal{P}_{1}(u)$.

Lemma 10. $L_{1}(u) \subseteq L_{0}(u)$.

Proof. Suppose to the contrary, that there are $p, p^{\prime} \in \mathcal{P}_{1}(u)$ such that $p-p^{\prime} \notin L_{0}(u)$. Then the sublattice $L_{1}^{\prime} \subseteq L$ generated by $p-p^{\prime}$ and by $L_{0}(u)$ has dimension $d$.

Consider the corresponding facet (commensurate) vectors $2 A p$ and $2 A p^{\prime}$. Since $p, p^{\prime} \in \mathcal{P}_{1}$, the facets $F(p)$ and $F\left(p^{\prime}\right)$ do not belong to the shadow boundary. Hence in the sum $P(a, \mathcal{P})+$ $z(u)$, these vectors are transformed into vectors $2 A p+u$ and $2 A p^{\prime}+u$. The difference of these vectors $2 A p-2 A p^{\prime}$ does not depend on the length of the vector $u$.

The lattice $2 A L$ contains the layers $2 A L_{0}(u), 2 A\left(L_{0}(u)+p\right)$ for $p \in \mathcal{P}_{1}(u)$ and $2 A\left(L_{0}(u)+\left(p-p^{\prime}\right)\right)$. The spacing between layers $2 A L_{0}(u)$ and $2 A L_{0}(u)+2 A\left(p-p^{\prime}\right)$ does not depend on the length of $u$. Call the last layer stationary. But the spacing between layers $2 A L_{0}(u)$ and $2 A\left(L_{0}(u)+p\right)$ for $p \in \mathcal{P}_{1}(u)$ do depend on the length of $u$. Chose a particular layer that moves and compare it with a layer that is stationary. Compare vertical heights of these layers by taking a ratio. The length of $u$ can be adjusted so that this ratio is irrational, in which case these layers do not generate a lattice. This is a contradiction.

Remark. Another proof of Lemma 10 can be found in Magazinov [4]. Referee asserts that Lemma 10 can be proved by using Theorem 6 of V'egh [8]. But Theorem 6 is not correct as it is stated.

Lemma 10 has the following important

Corollary 1. Let the Voronoi parallelotope be irreducible. Then, for any $p \in \mathcal{P}_{1}(u)$, the scalar product $\langle p, u\rangle=w>0$ does not depend on $p$. In other words, $u=$ we for some $e \in \mathcal{P}_{n}^{*}$, since $\langle p, u\rangle=0$ for $p \in \mathcal{P}_{0}(u)$ and $\mathcal{P}_{n}(a)=\mathcal{P}_{0}(u) \cup\left( \pm \mathcal{P}_{1}(u)\right.$.

Corollary 1 implies Theorem 4.

Now Theorems 3 and 4 imply

Theorem 5. Let $P(a, \mathcal{P})$ be an irreducible Voronoi parallelotope, defined in (1). Let $u \in \mathbb{R}^{d}$ be a vector. Then the following assertions are equivalent:

(i) Minkowski sum $P(a, \mathcal{P})+z(u)$ is a Voronoi parallelotope;

(ii) $u=$ we for some vector $e \in \mathcal{P}_{n}^{*}(a), z(u)=w P\left(a_{e}, \mathcal{P}\right)$.

Both the above conditions imply

$$
P(a, \mathcal{P})+z(u)=P(a, \mathcal{P})+w P\left(a_{e}, \mathcal{P}\right)=P\left(a+w a_{e}, \mathcal{P}\right)
$$

Theorem 5 is a generalization of results for Voronoi polytopes of root lattices $D_{n}, E_{6}$ and $E_{7}$ obtained in papers Grishukhin [10], Dutour at all [9] and Grishukhin [11], respectively.

Theorem 5 have the following important Corollary.

Corollary 2. If $\mathcal{P}_{n}^{*}(a)=\emptyset$, then $P(a, \mathcal{P})+z(u)$ is not a parallelotope for any vector $u$.

Examples of $P(a, \mathcal{P})$ with $\mathcal{P}_{n}^{*}(a)=\emptyset$ are Voronoi parallelotopes of lattices $E_{6}^{*}$ and $E_{7}^{*}$ that are dual of the root lattices $E_{6}$ and $E_{7}$ (see Dutour at al [9] and Grishukhin [11]).

Note that if the Voronoi parallelotope is reducible, then one can apply Theorem 5 to each component separately. This gives the following

Theorem 6. Let $P(a, \mathcal{P})$ be a Voronoi parallelotope, defined in (1). Let $P(a, \mathcal{P})=\sum_{i=1}^{k} P\left(a, \mathcal{P}_{i}\right)$, where sum is direct and $P\left(a, \mathcal{P}_{i}\right)$ is an irreducible parallelotope for each $i$. Let $\mathcal{P}_{\text {in }}(a)$ be a set of normal vectors of the parallelotope $P\left(a, \mathcal{P}_{i}\right)$ for all $1 \leq i \leq k$. Let $u \in \mathbb{R}^{d}$ be a vector. Then the following assertions are equivalent:

(i) Minkowski sum $P(a, \mathcal{P})+z(u)$ is a Voronoi parallelotope;

(ii) $u=\sum_{i=1}^{k} w_{i} e_{i}$, where $e_{i} \in \mathcal{P}_{i n}^{*}(a)$, and $z(u)=$ $\sum_{i=1}^{k} w_{i} P\left(a_{e_{i}}, \mathcal{P}\right)$.

Both the above conditions imply

$P(a, \mathcal{P})+z(u)=P(a, \mathcal{P})+\sum_{i=1}^{k} w_{i} P\left(a_{e_{i}}, \mathcal{P}\right)=P\left(a+\sum_{i=1}^{k} w_{i} a_{e_{i}}, \mathcal{P}\right)$.

Let $\mathcal{P}_{i 0}=\left\{p \in \mathcal{P}_{i n}:\langle p, u\rangle\right\}$ and let $L_{i 0}(u)$ be the lattice generated by vectors of $\mathcal{P}_{i 0}$. Let $d_{i}$ be dimension of $P\left(a, \mathcal{P}_{i}\right)$. 
By Lemma 9, $\operatorname{dim} L_{i 0}(u)=d_{i}-1$. The set $\cup_{i} \mathcal{P}_{i 0}(u)$ generates the lattice $\sum_{i} L_{i 0}(u)$ of dimension $\sum_{i=1}^{k}\left(d_{i}-1\right)=d-k$. This shows that dimension of the perfect space is less than $d-1$ if the parallelotope $P(a, \mathcal{P})$ is reducible.

\section{Acknowledgements}

The authors are deeply grateful to A.A.Gavrilyuk for fruitful discussions.

\section{REFERENCES}

[1] G.F.Voronoi, Nouvelles applications des paramètres continu à la théorie des formes quadratiques, J. für die Reine und Angewandte Mathematik, Vol. 133, 97-178, 1908; Vol. 134, 198-287, 1908; Vol. 136, 67-181, 1909.

[2] N.P.Dolbilin, Properties of faces of parallelohedra, Trudy MIAN, Vol. 266, 112-126, 2009.

[3] V.Grishukhin, Minkowski sum of a paralleltope with a segment, Math. Sbornik, Vol. 197, No. 10, 15-32, 2006 (translated in Sbornik: Math. Vol. 197, No. 10, 1417-1433, 2006).

[4] A. Magazinov, Voronoi's conjecture for extensions of Voronoi parallelohedra, 1-32, 2013 submitted to Discrete and Computational Geometry.
[5] S.S.Ryshkov, E.A.Bol'shakova, On the theory of mainstay parallelohedra, Izvestia: Math., Vol. 69, No. 6, 1257-1277, 2005.

[6] Á.G.Horváth, On the connection between the projection and the extension of a parallelotope, Monatshefte für Mathematik, Vol. 150, No 3, 211-216, 2007.

[7] B.A.Venkov, On projection of parallelohedra, Math. Sbornik Vol. 49, No. 2, 207-224, 1959 (in Russian).

[8] A.Végh, On extraction of parallelotopes, Stud. Univ. Zilina, Math. Ser. Vol. 26, 49-54, 2014.

[9] M.Dutour Sikirić, V.Grishukhin, A.Magazinov, On the sum of a parallelotope and a zonotope, Europ. J. Combinatorics Vol. 42, 49-73, 2014.

[10] V.Grishukhin, Free and non-free Voronoi polytopes, Mat. Zametki Vol. 80, No. 3, 367-378, 2006.

[11] V.Grishukhin, Delaunay and Voronoi polytopes of the root lattice $E_{7}$ and its dual $E_{7}^{*}$, Trudy MIAN Vol. 275, 68-86, 2011. (translated in Proceedings of Steklov Inst. of Math. Vol. 275, 60-77, 2011). 